\title{
Comparison of occupational burnout scores among medical residents and their correlation with dimensions of $\mathrm{TCl}$ questionnaire
}

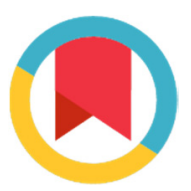

\author{
Mandana Haghshenas ${ }^{1}$, Ruohollah Seddigh ${ }^{2}$, Nasibeh Hashemkhani ${ }^{1}$, AmirAbbas Keshavarz-Akhlaghi ${ }^{2}$, Ahmad Mousavi $^{1}$, \\ Shiva Soraya*2
}

Received: 14 Oct 2018

Published: 6 Nov 2019

\section{Abstract}

Background: The present study was conducted to compare occupational burnout scores and determine their correlation with different dimensions of Temperament and Character Inventory (TCI) questionnaire among psychiatry, internal medicine, and surgery residents during the academic year 2013-14.

Methods: In this cross sectional analytical study, 201 residents were recruited. Colinger's 125-item TCI and Maslach's Burnout Inventory were completed by residents. The mean severity of burnout and the mean scores in the subgroups of temperament and character were compared between the 3 groups of residents, and the correlations were calculated. Data were analyzed using SPSS software version 16 . Also, A 2-sided $p$ value of less than 0.05 was considered significant.

Results: A significant positive correlation was found between severity of burnout and harm avoidance in internal medicine residents $(\mathrm{r}=0.7, \mathrm{p}<0.001)$. Also, a significant correlation was found between severity of burnout and self-directedness in surgery residents $(\mathrm{r}=0.5, \mathrm{p}=0.003)$, self-transcendence in internal medicine residents $(\mathrm{r}=0.04, \mathrm{p}=0.009)$, and persistence in internal medicine $(\mathrm{r}=0.17$, $\mathrm{p}=0.003)$ and surgery residents $(\mathrm{r}=0.10, \mathrm{p}=0.004)$. A significant correlation was found between frequency of burnout and harm avoidance in internal medicine residents $(\mathrm{r}=0.6, \mathrm{p}=0.001)$, self-directedness in surgery residents $(\mathrm{r}=0.9, \mathrm{p}<0.001)$, persistence in surgery $(\mathrm{r}=0.14, \mathrm{p}<0.001)$ and psychiatry residents $(\mathrm{r}=0.19, \mathrm{p}<0.001)$, and finally self-transcendence in internal medicine residents $(\mathrm{r}=0.6, \mathrm{p}<0.001)$.

Conclusion: Dimensions of character were different among surgery, internal medicine, and psychiatry residents. Likewise, the severity of burnout was different among them according to personality traits. Occupational burnout appears to be less if personality traits match the chosen specialty.

Keywords: Occupational burnout, Personality, Psychiatry, Internal, Surgery

Conflicts of Interest: None declared

Funding: None

*This work has been published under CC BY-NC-SA 1.0 license.

Copyright $\odot$ Iran University of Medical Sciences

Cite this article as: Haghshenas M, Seddigh R, Hashemkhani N, Keshavarz-Akhlaghi AA, Mousavi A, Soraya Sh. Comparison of occupational burnout scores among medical residents and their correlation with dimensions of TCI questionnaire. Med J Islam Repub Iran. 2019 (6 Nov);33:118. https://doi.org/10.47176/mjiri.33.118

\section{Introduction}

According to the World Health Organiza- tion (WHO), occupational burnout is a syndrome (group

Corresponding author: Dr Shiva Soraya, soraya.s@iums.ac.ir

1. School of Medicine, Iran University of Medical Sciences, Tehran, Iran

2. Spiritual Health Research Center, Iran University of Medical Sciences, Tehran, Iran $\uparrow$ What is "already known" in this topic:

Personality traits reflect the individual's mental ability /efficiency and should be addressed when guiding people to choose a career or field of study. Studies have shown that some people with high IQs are not suitable for certain fields of study or work because they lack certain personality traits and can instead achieve significant success in other academic /career environments.

\section{$\rightarrow$ What this article adds:}

Given the lack of similar studies and the need for research in this field, especially in Iran, and since the results of this study may be of use to help encourage the choice of a medical specialty in accordance with personal characteristics, the present study was conducted to examine and compare occupational burnout scores in medical residents with different specialties. 
of symptoms) linked to long-term, unresolved, and workrelated stress. On the one hand, this syndrome affects the mental health and well-being of the staff and, on the other, leads to some complications such as medical errors; also as the rate increases, so does the burnout $(1,2)$. Some studies inform about a direct relationship between burnout and patients' mortality $(3,4)$. In one study, $90 \%$ of the physicians had moderate to severe occupational burnout (5). Burnout is also prevalent in a significant percentage of residents of various medical disciplines (6). Among the various specialties, factors such as having more work pressure and being more exposed to the unwell and the elderly, chronic and incurable patients, and a state of urgency in their careers (7), feeling low trust from patients, long and continuous working hours, and higher psychological job demands (8), large numbers of patients, crowded emergency rooms, and individual parameters (coping mechanisms, social development, and work environment) (9) have a determining role in occupational burnout.

Since occupational burnout is related to occupational and demographic variables and varies across different personality groups, it can be concluded that certain personality traits, such as neuroticism, extroversion, flexibility, agreeableness, and conscientiousness, predict the rise or fall in the likelihood of occupational burnout. For instance, idealism, low resilience, poor self-esteem, external locus of control, and avoidance coping styles are associated with greater likelihood of burnout $(10,11)$. On the other hand, in an equal setting, people with certain personality traits, such as extroversion, flexibility, adaptation, and conscientiousness, have better work efficiency and greater job satisfaction compared to others (12). This also applies to progress in an academic discipline, meaning that in addition to intelligence and memory, personality traits are also among factors affecting learning (13). Circumstantial factors can both affect personality of an individual and reveal some of their attributes. In other words, certain personality traits may only be exhibited in particular circumstances (14). According to the personality theory of career choices, people choose a career according to their personality traits. People that choose the same jobs have similar personalities. John L Halland believes that job satisfaction is the compatibility of personality type with the working environment and that job satisfaction increases with increasing compatibility between them (15). Adaptation and harmony between personality and environment types leads to greater compatibility with the occupation, which in turn leads to occupational and personal satisfaction (15).

Job satisfaction is a highly important factor in career success and leads to enhanced efficiency and personal satisfaction. Job satisfaction refers to a kind of positive feeling toward working and results from several factors, such as workplace, organizational system, relationships, ruling workplace, cultural factors, and most importantly, nature of the job itself (15). Several studies have shown that in the same workplace with similar conditions, job satisfaction and efficiency are higher and burnout is lower in some people with certain attributes. However, these features vary in different occupations (16). Positive relationships were observed between certain personality traits and increased occupational burnout among nurses as a member of the medical team. It can, therefore, be concluded that inherent attributes of individuals make them suitable for certain jobs (12). Few studies have been conducted in this field, especially in Iran, and the present study results can be used to introduce measures toward increased job satisfaction, reduced burnout, and greater compatibility between personal characteristics and the chosen specialty by physicians. Therefore, this study aimed to compare burnout scores and assess the correlation between these scores and scores of 7 dimensions of Temperament and Character Inventory (TCI) among psychiatry, internal medicine, and surgery residents.

\section{Methods}

This cross sectional analytical study was designed using nonrandom convenient sampling method. The study population comprised 201 first, second, third, and fourth year psychiatry, internal medicine, and surgery residents from hospitals affiliated to Iran University of Medical Sciences during 2013-14 academic year. After obtaining the questionnaires from the Mental Health Research Center and School of Behavioral and Mental Health Sciences and after coordination with the chief of internal medicine, surgery, and psychiatry departments, questionnaires were made available to residents in related disciplines in all 4 academic levels in the morning report meeting. The questionnaires were collected after completion during the same session. If residents did not have enough time, questionnaires were collected during the morning report on the following day. Questionnaires included a demographic questionnaire, Colinger's 125-item TCI and Maslach's Burnout Inventory (MBI), which contains 22 items and measures severity of occupational burnout in 3 dimensions of depersonalization (DP), emotional exhaustion (EE), and diminished sense of personal accomplishment (PA). MBI items are scored based on a 7-point Likert scale. The period of 3 months was considered for data collection. Cronbach's alpha for EE, DP, and PA subscales was found to be $0.89,0.80$, and 0.76 , respectively. In the present study, all principles of Helsinki Declaration (ethical principles in medical research on humans) and ethical principles approved by the Ministry of Health were adhered to in all stages. Name and data obtained from residents remained confidential. No expenses were imposed on residents. Data were analyzed using SPSS software version 16. Also, one-sample KS was used to test the normality of distribution. Quantitative variables with normal distribution were reported using mean and standard deviation and qualitative variables were reported using frequency and percentage. Student $t$ test was used to compare the mean values of quantitative variables with normal distribution and chi-squared test was utilized to compare qualitative variables. Also, Pearson correlation coefficient (r) was used to measure the strength of linear relationship between the 2 variables. The frequency and severity of occupational burnout in residents of different disciplines were compared, and the correlation between scores of 
burnout and scores of dimensions of TCI was determined. One-way ANOVA test results showed no significant difference among residents in the mean scores of burnout. Furthermore, the distribution of different personality types among residents of various disciplines was reported elsewhere (17). A 2-sided $p$ value of less than 0.05 was considered significant.

1- Cloninger's TCI: TCI (Temperament and Character Inventory) is a set of tests for evaluating personality traits devised by Cloninger et al and consists of 7 basic personality dimensions of temperament and character: novelty seeking (NS), harm avoidance (HA), reward dependence (RD), persistence (PS), self-directedness (SD), cooperativeness (CO), and self-transcendence (ST). The 125-item self-report questionnaire of the TCI Cloning questioner has been validated and verified in 2005 by Kaviani and Purnaseh for Iranian population. The alpha coefficient for the reliability of the test retest in different subgroups of the questionnaire was between 0.73 and $0.90(p<0.001)$. The internal consistency between the scales of the questionnaire was between 0.55 and 0.84 , and the coefficients of the validity of the scale of the questionnaire was between 0.66 to $0.90(\mathrm{p}<0.001)$. $(18,19)$

2- MBI (GS): The Mazlach Burnout questionnaire (MBI) is a 22-item survey that covers 3 areas pertaining to occupational burnout, including emotional exhaustion, depersonalization, and diminished sense of personal accomplishment. The scoring of the items in this questionnaire is based on a 7-point Likert scale. Cronbach's alpha for EE, DP, and PA subscales is $0.89,0.80$, and 0.76, respectively (20). In Sepehri Shamlo et al study on an Iranian population, internal consistency, evaluated by coefficient Cronbach's Alpha, was 0.87 and the total correlation was measured to be 0.79 and 0.85 , which is considered as a high reliability (21). Measuring test-retest reliability showed an interclass correlation coefficient of 0.87 , which is a good test-retest reliability $(\mathrm{r}=0.87, \mathrm{p}<0.01)$. Then, the Kaiser-Meyer-Olkin (KMO) and Bartlett's test were used to ensure the appropriateness of data to be used in this method. KMO test value was $0.865(\mathrm{KMO}<0.05)$ and the Bartlett's test value was equal to $2393.89(\mathrm{p}=0.01)$, indicating suitability of data for factor analysis. The construct validity of the scale based on Kaiser Criteria (eigenvalues greater than 1) showed 3 factors with eigenvalues greater than 1 ( 1 and 5 items: $\alpha=0.72 ; 2,4$ items: $\alpha=0.78 ; 3,6$ items: $\alpha=0.69)$. However, 3 -factor specification was the best. In confirmatory factor analysis, the original 3-factor model of MBI-GS was adequate $\left(\mathrm{X}^{2} / \mathrm{df}=582.9 / 74\right.$; goodness-of-fit index $=0.86$; root mean square error of approximation $=0.05$; and comparative fit index $=0.89)(21,22)$.

\section{Results}

In this study, $49.8 \%$ of the respondents were female $(n=100)$ and $50.2 \%$ were male $(n=101)$. Also, 35.8\% $(n=72)$ were internal medicine residents, 35.3\% $(n=71)$ surgery residents, and $28.9 \%(n=58)$ psychiatry residents. Moreover, $45.8 \%(n=92)$ of the participants were single and $54.2 \%(n=109)$ were married. No significant relationship was observed between gender and academic discipline. Severity and frequency of burnout were different in residents of different disciplines. According to Maslach questionnaire, the mean score of occupational burnout was $2.22 \pm 0.51$ in internal medicine residents, $2.17 \pm 0.52$ in surgery residents, and $2.14 \pm 0.58$ in psychiatry residents, No significant difference was observed among different residents in frequency of burnout. Figures 1 to 3 show the frequency of burnout in internal medicine, surgery, and psychiatry residents. One-way ANOVA test results showed no significant difference among residents in the mean scores of burnout $(\mathrm{p}=0.664)$.

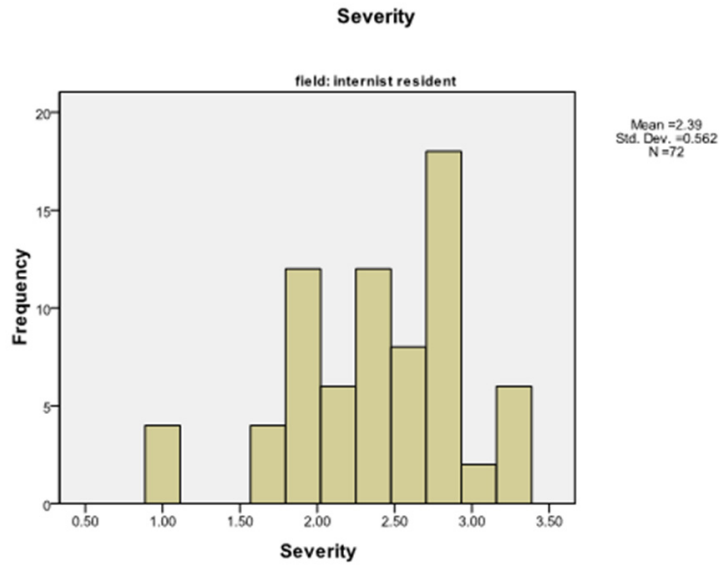

Fig. 1. Frequency distribution of burnout in internal medicine Residents

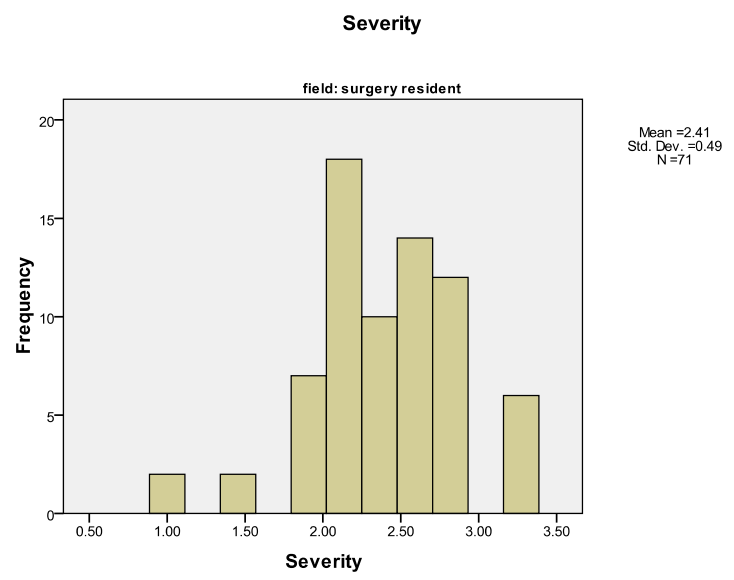

Fig. 2. Frequency distribution of burnout in surgery residents

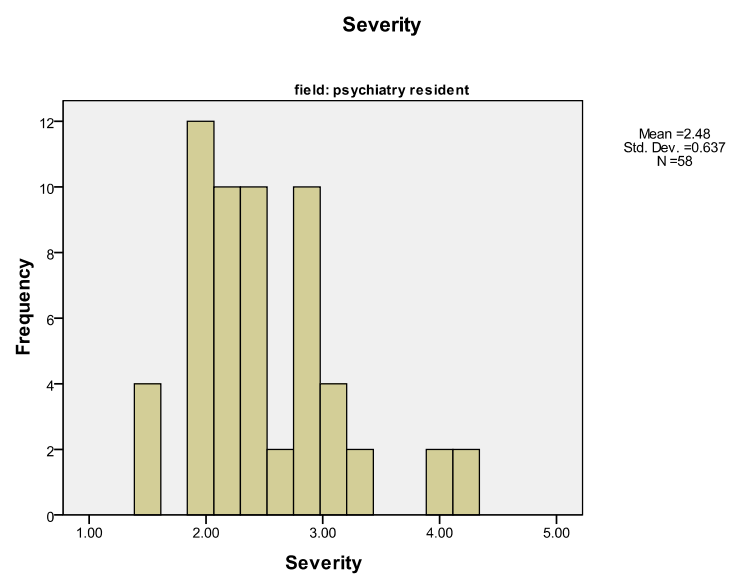

Fig. 3. Frequency distribution of burnout in psychiatry residents 


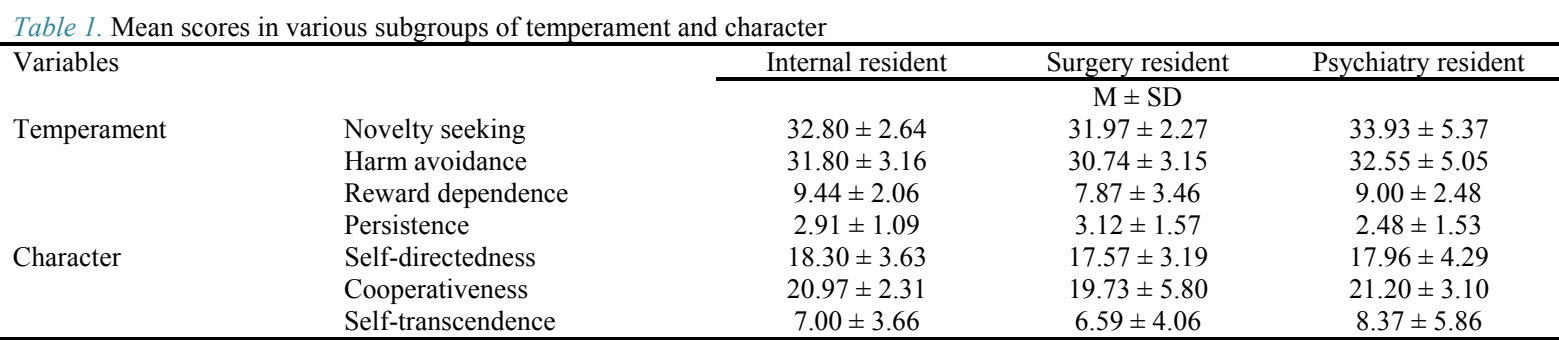

The mean severity of burnout was $2.39 \pm 0.56$ in internal medicine residents, $2.41 \pm 0.49$ in surgery residents, and $2.48 \pm 0.63$ in psychiatry residents, with zero indicating none of the emotions mentioned in the questionnaire, 4 moderate severity, and 7 extremely severe feelings. Oneway ANOVA test results revealed no significant difference among residents in severity of burnout $(p=0.631)$. The mean scores of internal medicine, surgery, and psychiatry residents in various subgroups of temperament and character scales of Colinger's TCI are presented in Table 1.

Pearson's correlation coefficient test results showed a significant positive correlation between severity of occupational burnout and harm avoidance in internal medicine residents $(r=0.7, p<0.001)$, a significant correlation between severity of burnout and self-directedness in surgery residents $(\mathrm{r}=0.5, \mathrm{p}=0.003)$, a significant correlation between severity of burnout and persistence in internal medicine $(r=0.17, p=0.003)$ and surgery residents $(r=0.10$, $\mathrm{p}=0.004$ ), and a significant correlation between severity of burnout and self-transcendence in internal medicine residents $(\mathrm{r}=0.04, \mathrm{p}=0.009)$.

Other dimensions of personality had no significant correlation with severity of burnout in residents $(p>0.05)$.

Pearson's correlation test results showed a significant correlation between frequency of burnout and harm avoidance in internal medicine residents $(r=0.6, p=0.001)$, a significant correlation between frequency of burnout and self-directedness in surgery residents $(r=0.9, \mathrm{p}<0.001)$, a significant correlation between frequency of burnout and persistence in surgery $(\mathrm{r}=0.14, \mathrm{p}<0.001)$ and psychiatry $(\mathrm{r}=0.19, \mathrm{p}<0.001)$ residents, and a significant correlation between frequency of burnout and self-transcendence in internal medicine residents $(\mathrm{r}=0.6, \mathrm{p}<0.001)$.

\section{Discussion}

This study investigated the relationship between personality traits and occupational burnout. Similar to those reported by Mirzabeig et al in Rafsanjan hospital, gender did not have a significant association with occupational burnout (5).

In a study by Martini et al, mean burnout was reported $63 \%$ in internal medicine residents and $40 \%$ in psychiatry and surgery residents. Although in the present study, the frequency of burnout was higher in internal medicine residents, the severity of burnout was higher in psychiatry residents (23). Internal medicine residents work greater number of shifts and longer hours in a stressful environment and have little control over the environment and work schedule. Most of the time, they provide care for patients in critical conditions in hospitals. Despite several diagnostic tests and various medical techniques, care is more extensive and residents may still lack perfect patient care ability, and this causes occupational stress and affects their quality of life. In a study conducted by Olkinuora et al (24) and Graham et al (25), risk and burnout factors were higher among surgeons compared to other specialists. According to Olkinuora theory, those specialists who deal with incurable, chronic, and dying patients are at greater risk of burnout than those who deal with patients with good prognosis. Another main factor affecting occupational burnout is considered psychological pressure caused by huge workload and long working hours. A significant positive correlation was found between the severity and frequency of burnout and harm avoidance in internal medicine residents. Melchers also reported a medium to large positive correlation between harm avoidance and burnout (26). Patients treated by internal medicine residents usually have more complex conditions and require selection of a test out of several diagnostic tests and treatment method out of many available methods. Internal medicine residents with greater harm avoidance experience higher levels of stress in such conditions. A significant correlation was also found between the severity and frequency of burnout and self-directedness in surgery residents, which is contrary to expectations. People with high self-directedness and ability to regulate and adjust behavior according to various situations are expected to have better management skills in difficult circumstances and lower severity of burnout. Further studies are recommended to find the reasons for this issue. As seen in Melchers' article, self-directedness and cooperativeness were negatively related to burnout (26). In Lee's study, lower scores of cooperativeness and self-directedness and higher scores of novelty-seeking and harm avoidance were correlated with greater academic burnout of medical students regardless of sex and age (27), which is different from the cases of self-directedness and novelty-seeking in the present study.

Significant correlations were observed between the severity of burnout and persistence in internal medicine residents and between the severity and frequency of burnout and persistence in surgery residents. Internal medicine and surgery residents with higher persistence are at greater risk for severity of burnout, but there is no such a correlation in psychiatry residents. A significant correlation was found between the severity and frequency of burnout and self-transcendence in internal medicine residents. However, in Mechler's study, persistence and self-transcendence revealed very small correlations to burnout (26). The reason for this difference may be the difference in the popu- 
lation studied in the Mechler's study, consisting German employees from a wide range of professions and psychiatry patients.

\section{Problems and limitations}

- Although the residents were not interested in participating in the study, after explaining the purpose of the study and its importance, they agreed to participate.

- Flawed completion of the questionnaire: The estimation for gathering correct and complete questionnaires was about $80 \%$, which was considered to be likely in this sample size. On the other hand, some incomplete questionnaires also extracted some personality traits. A questionnaire filled with all its options in one form (for example, all responses in option A) or questionnaires that were associated with a specific pattern of response were deleted.

Dimensions of personality were different in surgery, internal medicine, and psychiatry residents, and the severity of burnout was also varied in residents of different disciplines according to their personality attributes. Thus, physicians do not choose their specialty disciplines merely based on personality attributes, and many other factors affect their choice, such as prospect of a job, preferred quality of life, social standing of the discipline, and compatibility of discipline with culture, society, and sometimes gender. However, higher compatibility between personality attributes and the chosen specialty discipline will increase the efficiency and job satisfaction and will reduce the possibility of occupational burnout.

\section{Conflict of Interests}

The authors declare that they have no competing interests.

\section{References}

1. Klein J, Grosse FK, Blum K, von dem Knesebeck O. Burnout and perceived quality of care among German clinicians in surgery. Int $\mathrm{J}$ Qual Health Care. 2010; 22:525-30.

2. Williams ES, Manwell LB, Konrad TR, Linzer M. The relationship of organizational culture, stress, satisfaction, and burnout with physician-reported error and suboptimal patient care: results from the MEMO study. Health Care Manag Rev. 2007;32(3):203-12.

3. Kane RL, Shamliyan TA, Mueller C, Duval S, Wilt TJ. The association of registered nurse staffing levels and patient outcomes: Systematic review and meta-analysis. Med Care. 2007;45:1195204.

4. Dall'Ora C, Ball J, Recio-Saucedo A, Griffiths P. Characteristics of shift work and their impact on employee performance and wellbeing: A literature review. Int J Nurs Stud. 2016;57:12-27.

5. Mirzabeigi M, Agha Mohammad Hasani P, Sheikh-Fathollahi M, Mokhtaree M. Correlation between occupational burnout and personality dimensions among physicians working in hospitals of Rafsanjan University of Medical Sciences, Iran, 2016. JOHE. 2018;7(3):153-9. (in Persian)

6. Bagheri SH, Kousha A, Janati A. Factors affecting nurses' job satisfaction: a systematic survey. Hakim. 2012;15:130-9. (Persian)

7. Atef L, Rooh-al-Amin M, Noori A, Molavi H. A comparison of job burnout in general surgeons and internists in Isfahan. Know Res Appl Psychol. 2006;8:129-51.

8. Huang EC, Pu C, Huang N, Chou YJ. Resident burnout in Taiwan Hospitals and its relation to physician felt trust from patients. J Formos Med Assoc. 2019.

9. Popa F, Raed A, Lorin Purcărea V, Lală A, Bobirnac G, Davila C. Occupational Burnout levels in Emergency Medicine - a nationwide study and analysis. J Med Life. 2010;3:207-15.

10. Hudek-Knežević J, Kalebić Maglica B, Krapić N. Personality, or- ganizational stress, and attitudes toward work as prospective predictors of professional burnout in hospital nurses. Croatian Med J. 2011;52:538-49.

11. Rasoulian M, Elahi F, Afkham Ebrahimi A. The relationship between job burnout and personality traits in nurses. J Indust Psychol. 2003;29:35-42.

12. Magalhães E, Costa P, Costa MJ. Empathy of medical students and personality: Evidence from the Five-Factor Model. Med Teach. 2012;34:807-12.

13. Ghorbani A, Noroozi K, Jahani-Hashem H. Correlation between type of personality and academic achievement of nursing students in university of medical sciences Qazvin. Iran J Nurs Res. 2013;8:24-31.

14. De Fruyt F, Van De Wiele L, Van Heeringen C. Cloninger's psychobiological model of temperament and character and the five-factor model of personality. Person Indiv Diff. 2000;29:441-52.

15. Shafie Abadi A. Guidance and career counseling. 1st ed. Tehran, Iran: Samt; 1999. (Persian)

16. Lounsbury JW, Park SH. Personality, career satisfaction, and life satisfaction: Test of a directional model. J Career Assess. 2004; 12:395-406.

17. Hashemkhani N, Sedigh RA, Keshavarz AA, Soraya SH. Comparison temperament and character in psychiatric, internal and surgery residents of Iran University of Medical Sciences in 1392-1393. Med J Islam Repub Iran. 2018. (in Press)

18. Cloninger CR. The temperament and character inventory (TCI): A guide to its development and Use. St. Louis, MO: Center for Psychology and Personality, Washington University; 1994.

19. Kaviani H, Poor Naseh M. Validation of temperament and character inventory (TCI) In Iranian sample: Normative data. Tehran Uni Med J. 2005;63:89-98.

20. Boles JS, Dean DH, Ricks JM, Short JC, Wang G. The dimensionality of the Maslach Burnout Inventory across small business owners and educators. J Voc Behav. 2000;56:12-34.

21. Sepehri Shamloo Z, Hashemian S S, Khoshsima H, Shahverdi A, Khodadost M, et al. Validity and Reliability of the Persian Version of the Maslach Burnout Inventory (General Survey Version) in Iranian Population. Iran J Psychiatry Behav Sci. 2017;11.

22. Maslach C, Jackson SE. The measurement of experienced burnout. J Occup Behav. 1981; :99-113.

23. Martini S, Arfken CL, Churchill A. Burnout comparison among residents in different medical specialties. Acad Psych. 2004;28:240 2.

24. Olkinuora M, Asp S, Juntunen J, Kauttu K, Strid L, Aärimaa M. Stress symptoms burnout and suicidal thoughts in finish physicians. Soc Psych Psych Epidemiol 1990; 25:81-6.

25. Graham J, Ramirez AJ, Cull A, Richards MA. A user's guide to the consultants mental health questionnaire. London: Guy's Hospital. 1996

26. Melchers MC, Plieger T, Meermann R, Reuter M. Differentiating burnout from depression: Personality matters!. Front Psych. 2015;6:113.

27. Lee SJ, Choi YJ, Chae H. The effects of personality traits on academic burnout in Korean medical students. Integr Med Res. 2017;6:207-13. 